\title{
Synthesis of new chiral alkenyl Fischer carbene complexes
}

\author{
José Barluenga*, Narendra Panday, Javier Santamaría, Ana de Prado, and Miguel Tomás \\ Instituto Universitario de Química Organometálica “E. Moles”. Unidad Asociada al CSIC \\ Universidad de Oviedo, Julián Clavería, 8, E-33006 Oviedo, Spain \\ E-mail: barluenga@sauron.quimica.uniovi.es
}

\section{Dedicated to Professor Edmundo A. Rúveda on the occasion of his $70^{\text {th }}$ birthday and to Professor Roberto A. Rossi on occasion of his $60^{\text {th }}$ birthday}

(received 12 Oct 03; accepted 17 Dec 03; published on the web 27 Dec 03)

\begin{abstract}
New chiral group 6 Fischer alkoxycarbene complexes $\mathbf{1}$ and $\mathbf{2}$ have been synthesized by condensation of enantiopure aldehydes $\mathbf{6}$ and 7, respectively, with methylcarbene complexes 5 . The corresponding aminocarbene complexes $\mathbf{3}$ and $\mathbf{4}$ were accessible from $\mathbf{1}$ and $\mathbf{2}$ by methoxypyrrolidine exchange. The chromium carbene $\mathbf{1 b}$ underwent the benzannulation reaction with alkynes affording chiral substituted phenols 11.
\end{abstract}

Keywords: Alkenyl Fischer carbene complexes, chromium, tungsten, chirality, (D)-glyceraldehyde, benzannulation

\section{Introduction}

Since their discovery by Fischer ${ }^{1}$ et al. in 1964, Fischer carbene complexes have emerged as powerful organometallic reagents in organic synthesis. ${ }^{2}$ Among the Fischer carbene complexes, $\alpha, \beta$-unsaturated carbenes have been demonstrated to be particularly attractive for synthetic purposes because of their polyfunctional and versatile character. ${ }^{3}$

On the other hand, the development of asymmetric processes based on chiral alkenyl Fischer carbene complexes have received increasing attention. ${ }^{4}$ Although incorporation of the chiral auxiliary at the ligands, e.g. chiral phosphines ${ }^{5}$ and phosphites, ${ }^{6}$ appears to be the ideal strategy for asymmetric synthesis, it has not been successfully implemented as a practical protocol. On the contrary, a number of efficient enantioselective syntheses using group 6 metal carbene complexes derived from chiral alcohols, ${ }^{7}$ amines, ${ }^{8}$ aminoalcohols, ${ }^{9}$ oxazolines, ${ }^{10}$ etc. have been reported to provide rapid access to optically active open-chain and cyclic molecules. ${ }^{11}$ Finally, carbene complexes with attached sugars are being studied by the group of K. H. Dötz. ${ }^{12}$ 
Here we report the synthesis of new chiral alkenyl Fischer carbene complexes 1-4 (Figure 1) derived from optically active reagents that are readily available from the natural chiral pool. The presence of the chiral center at the $\beta$-position is expected to induce high selectivity, especially in the case of reactions initiated at that position. Moreover, this inductive effect would complement that provided at the carbene carbon by the chiral alkoxy group in carbene complexes derived from chiral alcohols.
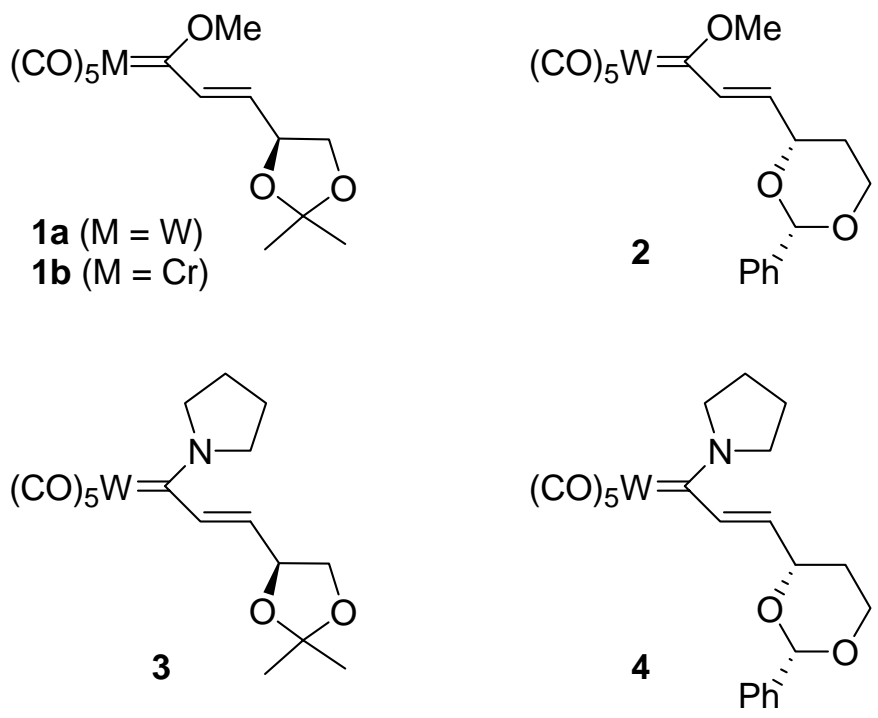

Figure 1

\section{Results and Discussion}

Alkoxycarbene complexes 1 and 2 were synthesized by condensation of the pentacarbonyl[methoxy(methyl)]carbene complexes 5 and aldehydes 6 and 7 (Figure 2). Aldehydes 6 and 7 were readily prepared from commercially available 1,2:5,6-diisopropylideneD-mannitol ${ }^{13}$ and (S)-malic acid ${ }^{14}$, respectively, following well known procedures.

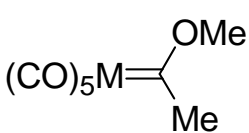

$5 a-b$

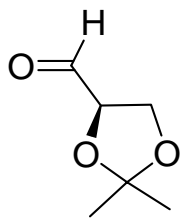

6

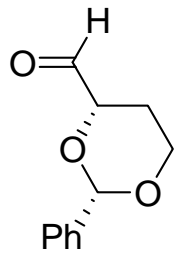

7

Figure 2

The condensation of enolizable aldehydes with $\alpha$-deprotonated carbene complexes is more problematic than with non-enolizable ones ${ }^{15}$ and usually requires a Lewis acid. ${ }^{16}$ Moreover, the rapid polymerization of aldehydes $\mathbf{6 , 7}$ prevents the use of Lewis acids. The synthesis of the 
alkoxyalkenylcarbene complexes 1,2 was actually achieved by diazabicycloundecane (DBU) deprotonation of the carbenes $\mathbf{5}$, followed by stirring at $0^{\circ} \mathrm{C}$ with freshly distilled aldehydes $\mathbf{6}$ or 7. The diastereomeric mixtures of $\mathbf{8}$ and $\mathbf{9}$, respectively, thus obtained were not isolated but reacted with methanesulfonyl chloride and triethylamine to induce the $\alpha, \beta$-elimination and to provide the desired carbene complexes 1a,b and $\mathbf{2}$ (Scheme 1).
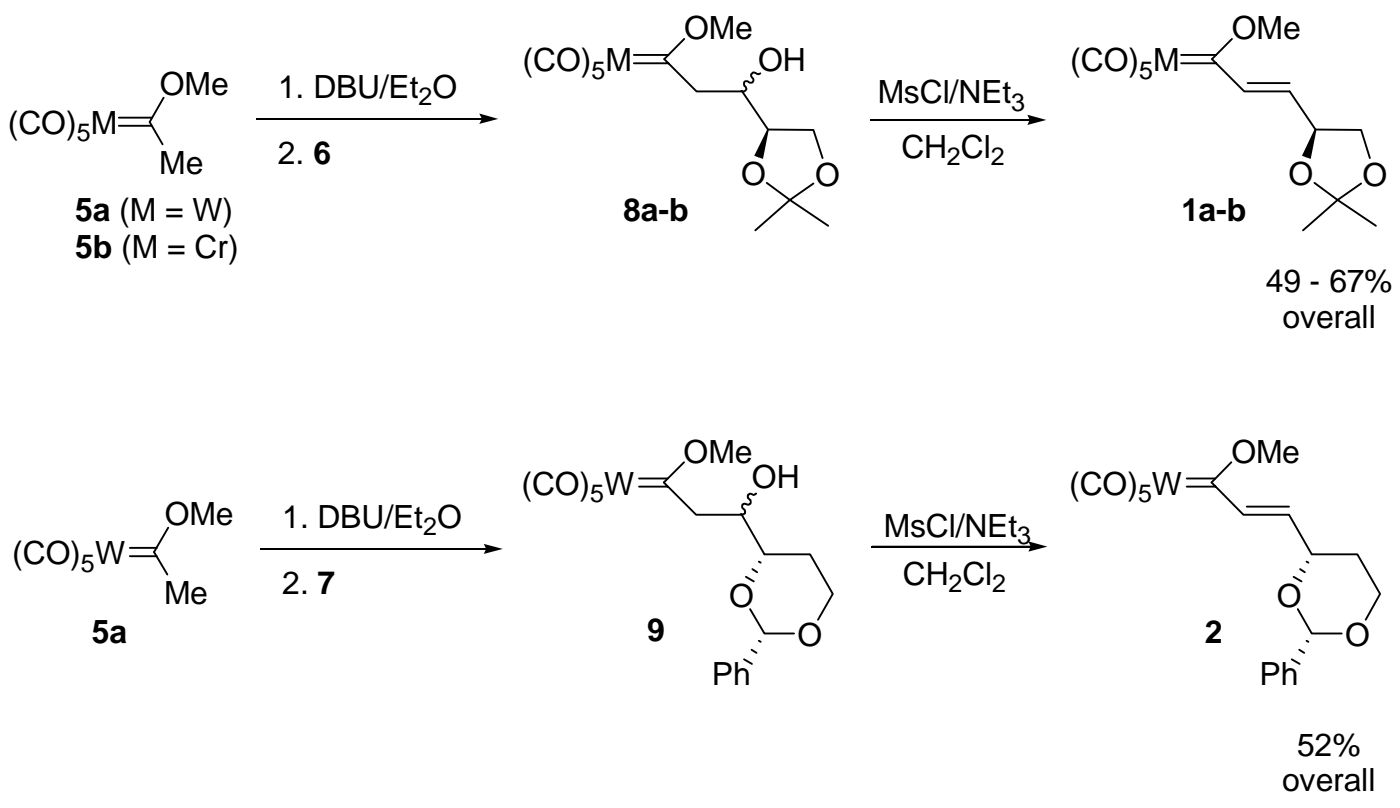

Scheme 1. Synthesis of the alkoxycarbenes 1,2 .

In order to determine the reactivity of the new Fischer carbene complexes, the alkoxy-amine exchange and the relevant Dötz benzoannulation ${ }^{17}$ reactions were studied. First, the alkenyl(methoxy)carbene complexes 1a and $\mathbf{2}$ smoothly reacted with pyrrolidine to afford new chiral aminocarbene complexes of chromium and tungsten $\mathbf{3}$ and $\mathbf{4}$, respectively, in good yields (Scheme 2).

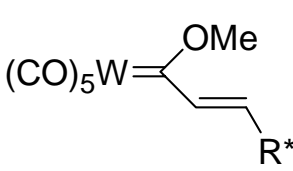

$1 \mathrm{a}, 2$

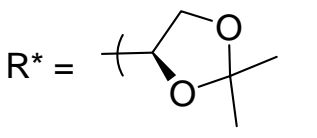

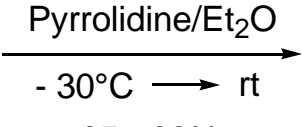

$85-88 \%$

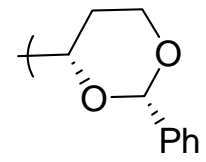<smiles>[R7]C=CC(=NC(C)(C)C)N1CCCC1</smiles>

3,4

Scheme 2. Synthesis of the aminocarbenes $\mathbf{3 , 4}$. 
It was satisfying to find that the chromium carbenes are as reactive as conventional analogs toward alkynes, being appropriate chiral organometallic reagents for the benzannulation reaction. Thus, heating the carbene complex $\mathbf{1 b}$ with alkynes $10 \mathbf{a}, \mathbf{b}$ at $60^{\circ} \mathrm{C}$ resulted in the regioselective formation of the chiral substituted phenols 11a,b in acceptable yields (Scheme 3). Apart from their importance as optically active materials, interesting applications, for instance as tripod-type ligands, ${ }^{18}$ can be envisioned for these cycloadducts due to the presence of various differentiable hydroxy functionalities.

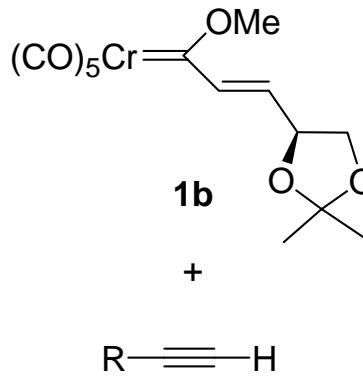

$10 \mathrm{a} \mathrm{R}=t-\mathrm{Bu}$

10b $\mathrm{R}=n-\mathrm{Bu}$

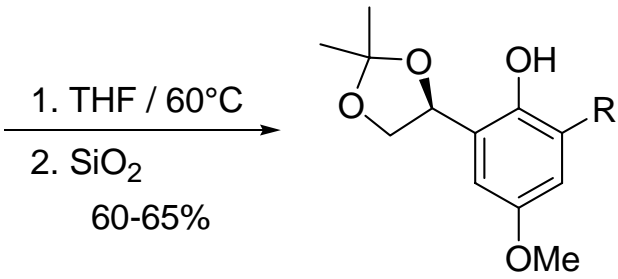

$11 a, b$

Scheme 3. Dötz benzannulation of the chiral carbene $\mathbf{1 b}$ with alkynes.

\section{Conclusions}

We have described a convenient synthesis of a new type or chiral pentacarbonylalkenylalkoxy and amino Fischer carbene complexes from easily accessible reagents. The new complexes appear to be reactive through either the carbene carbon or the $\beta$-carbon positions. Further applications of these complexes in enantioselective synthesis are being performed in our laboratory and will be reported in the near future.

\section{Experimental Section}

General Procedures. All reactions and purifications, except 11, were carried out under an atmosphere of dry $\mathrm{N}_{2}$. TLC was performed on aluminium-backed plated coated with silica gel 60 with $\mathrm{F}_{254}$ indicator (Merck). Flash column chromatography was carried out, under nitrogen atmosphere, on silica gel 60 and solvents used for the chromatographic purification of the carbene complexes was previously deoxygenated and saturated with $\mathrm{N}_{2} \cdot{ }^{1} \mathrm{H}\left({ }^{13} \mathrm{C}\right)$-NMR spectra were recorded at 200 (50) or 300 (75) MHz on Brucker instruments. High-resolution mass spectra (HRMS) were performed on a Finnigan MAT 95 spectrometer. All commercially available reagents were used without purification unless otherwise indicated. 
General procedure for the synthesis of alkoxycarbenes $(1,2)$. Pentacarbonyl carbene complex 5 (4 mmol) and freshly synthesized aldehydes 6,7 (4.5 mmol) were dissolved in $20 \mathrm{ml}$ of dry THF and cooled to $0^{\circ} \mathrm{C}$. To this solution, $50 \mu \mathrm{l}$ of DBU were added, the mixture stirred at $0^{\circ} \mathrm{C}$ (30 min for 1 and 90 min for 2 ) and the solvents removed under vacuo ( $10^{-2}$ torr.). The residue was dissolved in $\mathrm{CH}_{2} \mathrm{Cl}_{2}(30 \mathrm{ml})$ and treated at $0^{\circ} \mathrm{C}$ with $\mathrm{NEt}_{3}(4 \mathrm{mmol}, 560 \mu \mathrm{l})$ and $\mathrm{MsCl}(4$ mmol, $315 \mu \mathrm{l})$. The mixture was stirred for only 5 minutes. The solvent was removed under vacuo $\left(10^{-2}\right.$ torr.) and the residue subjected to chromatographic purification to obtain the alkolxycarbenes $\mathbf{1 , 2}$ as red oils.

Pentacarbonyl[E-3-((S)-2,2-dimethyl-1,3-dioxolan-4-yl)-1-methoxy-2-propenyliden]tungsten(0) (1a). Yield: 67\%; $\mathrm{R}_{\mathrm{f}}(\mathrm{Hex} / \mathrm{AcOEt}(10: 1))=0.41$; HRMS calculated for $\mathrm{C}_{14} \mathrm{H}_{14} \mathrm{O}_{8} \mathrm{~W}$ : 494.0193; found: $494.0184 ;{ }^{1} \mathrm{H}-\mathrm{NMR}\left(200 \mathrm{MHz}, \mathrm{CDCl}_{3}\right) \delta(\mathrm{ppm})=1.45$ (s, 3H), 1.5 (s, 3H), 3.7 (dd, $J=$ 8.2 and $7.2 \mathrm{~Hz}, 1 \mathrm{H}$ ), 4.2 (dd, $J=8.2$, and $6.7 \mathrm{~Hz}, 1 \mathrm{H}), 4.55-4.65$ (m, 1H), 4.6 (s, 3H), 6.3 (dd, $J=15.1$ and $5.9 \mathrm{~Hz}, 1 \mathrm{H}), 7.4(\mathrm{dd}, J=15.1$ and $0.8 \mathrm{~Hz}, 1 \mathrm{H}) ;{ }^{13} \mathrm{C}-\mathrm{NMR}\left(50 \mathrm{MHz}, \mathrm{CDCl}_{3}\right)$ : $\delta(\mathrm{ppm})=25.6(\mathrm{q}), 26.3(\mathrm{q}), 68.6(\mathrm{t}), 69.1(\mathrm{q}), 75.1(\mathrm{~d}), 110.3(\mathrm{~s}), 133.2(\mathrm{~d}), 146.6(\mathrm{~d}), 197.0(\mathrm{~s})$, 198.3 (s), 310.3 (s).

Pentacarbonyl[E-3-((S)-2,2-dimethyl-1,3-dioxolan-4-yl)-1-methoxy-2-propenyliden]chromium(0) (1b). Yield: 49\%. $\mathrm{R}_{\mathrm{f}}(\mathrm{Hex} / \mathrm{AcOEt}(5: 2))=0.43 ;{ }^{1} \mathrm{H}-\mathrm{NMR}\left(200 \mathrm{MHz}, \mathrm{CDCl}_{3}\right.$ ) $\delta$ (ppm)= 1.45 (s, 3H), 1.5 (s, 3H), 3.7 (dd, $J=8.2$ and $6.9 \mathrm{~Hz}, 1 \mathrm{H}), 4.2$ (dd, $J=8.5$ and $6.9 \mathrm{~Hz}, 1 \mathrm{H}), 4.5-4.7$ (m, $1 \mathrm{H}), 4.8$ (s, 3H), 6.05 (dd, $J=15.1$ and $5.9 \mathrm{~Hz}, 1 \mathrm{H}$ ), 7.5 (dd, $J=15.1$ and $1.3 \mathrm{~Hz}, 1 \mathrm{H}$ ); ${ }^{13} \mathrm{C}-\mathrm{NMR}$ $\left(50 \mathrm{MHz}, \mathrm{CDCl}_{3}\right): \delta(\mathrm{ppm})=25.7(\mathrm{q}), 26.4(\mathrm{q}), 66.6(\mathrm{q}), 69.0$ (t), 75.0 (q), 110.4 (s), 129.1 (d), 142.8 (d), 216.3 (s), 223.9 (s), 337.3 (s).

Pentacarbonyl[1-methoxy-3-((2S,4S)-2-phenyl-1,3-dioxan-4-yl)-2-propenyliden]tungsten(0) (2). Yield: 52\%. $\mathrm{R}_{\mathrm{f}}(\mathrm{Hex} / \mathrm{AcOEt}(10: 1))=0.30$; HRMS calculated for $\mathrm{C}_{19} \mathrm{H}_{16} \mathrm{O}_{8} \mathrm{~W}$ : 556.0349; found: 556.0311; ${ }^{1} \mathrm{H}-\mathrm{NMR}\left(200 \mathrm{MHz}, \mathrm{CDCl}_{3}\right) \delta(\mathrm{ppm})=1.7(\mathrm{dq}, J=13.1$ and $1.5 \mathrm{~Hz}, 1 \mathrm{H}), 2.0$ (dq, $J=13.8$ and $4.6 \mathrm{~Hz}, 1 \mathrm{H}$ ), 4.05 (dt, $J=11.8$ and $2.9 \mathrm{~Hz}, 1 \mathrm{H}$ ), 4.35 (dd, 12.8 and $6.4 \mathrm{~Hz}, 1 \mathrm{H}$ ), $4.5(\mathrm{~m}, 1 \mathrm{H}), 4.6(\mathrm{~s}, 3 \mathrm{H}), 5.6(\mathrm{~s}, 1 \mathrm{H}), 6.4(\mathrm{dd}, J=15.4$ and $4.9 \mathrm{~Hz}, 1 \mathrm{H}), 7.3-7.6(\mathrm{~m}, 6 \mathrm{H})$; ${ }^{13} \mathrm{C}-$ NMR $\left(75\right.$ MHz, $\left.\mathrm{CDCl}_{3}\right) \delta(\mathrm{ppm})=30.6(\mathrm{t}), 66.6(\mathrm{t}), 69.1$ (d), 75.6 (q), 100.9 (d), $125.9(\mathrm{~d}), 128.2$ (d), 128.9 (d), 135.5 (d), 138.1 (s), 145.5 (d), 197.2 (s), 203.6 (s), 311.0 (s).

\section{General procedure for the synthesis of the aminocarbenes 3, 4}

A solution of alkoxycarbenes 1,2 (2 mmol) in $20 \mathrm{ml}$ of dry diethyl ether was cooled to $-30^{\circ} \mathrm{C}$ and pyrrolidine (2,2 mmol, $160 \mathrm{mg}$ ) was added. The mixture was removed from the cooling bath and stirred at room temperature for 30 minutes. Removal of the solvent under vacuo (10 2 torr.) and chromatographic purification yielded the aminocarbene complexes 3,4 as yellow oils.

Pentacarbonyl[E-3-((S)-2,2-dimethyl-1,3-dioxolan-4-yl)-1-( $N$-pyrrolidinyl)-2propenyliden]tungsten(0) (3). Yield: $88 \%$; $\mathrm{R}_{\mathrm{f}}(\mathrm{Hex} / \mathrm{AcOEt}(1: 1))=0.53$; HRMS calculated for $\mathrm{C}_{17} \mathrm{H}_{19} \mathrm{NO}_{7} \mathrm{~W}: 533.0665$; found: 533.0661; ${ }^{1} \mathrm{H}-\mathrm{NMR}\left(300 \mathrm{MHz}, \mathrm{CDCl}_{3}\right) \delta(\mathrm{ppm})=1.4(\mathrm{~s}, 3 \mathrm{H})$, $1.45(\mathrm{~s}, 3 \mathrm{H}), 2.1(\mathrm{~m}, 4 \mathrm{H}), 3.6(\mathrm{~m}, 3 \mathrm{H}), 4.0(\mathrm{~m}, 2 \mathrm{H}), 4.2(\mathrm{dd}, J=8.1$ and $6.5 \mathrm{~Hz}, 1 \mathrm{H}), 4.6(\mathrm{q}, J=$ $6.7 \mathrm{~Hz}, 1 \mathrm{H}), 5.3(\mathrm{dd}, J=16.1$ and $6.4 \mathrm{~Hz}, 1 \mathrm{H}), 6.6(\mathrm{~d}, J=16.1 \mathrm{~Hz}, 1 \mathrm{H}) ;{ }^{13} \mathrm{C}-\mathrm{NMR}(75 \mathrm{MHz}$, 
$\left.\mathrm{CDCl}_{3}\right) \delta(\mathrm{ppm})=$ 24.7(t), $25.4(\mathrm{t}), 25.7(\mathrm{q}), 26.4(\mathrm{q}), 53.8(\mathrm{t}), 61.5(\mathrm{t}), 69.4(\mathrm{t}), 75.8(\mathrm{~d}), 109.5$ (d), 123.8 (d), 142.4 (d), 198.7 (s), 205 (s), 250 (s).

Pentacarbonyl[E-3-(2S,4S)-2-phenyl-1,3-dioxan-4-yl-1-( $N$-pyrrolidinyl)-2propenyliden]tungsten( (0) (4). Yield: 85\%; $\mathrm{R}_{\mathrm{f}}(\mathrm{Hex} / \mathrm{AcOEt}(5: 1))=0.18$; HRMS calculated for $\mathrm{C}_{22} \mathrm{H}_{21} \mathrm{NO}_{7} \mathrm{~W}$ : 595.0822; found: 595.0837; ${ }^{1} \mathrm{H}-\mathrm{NMR}\left(\mathrm{CDCl}_{3}, 300 \mathrm{MHz}\right) \delta(\mathrm{ppm})=1.7$ (d, $J=$ $11.9 \mathrm{~Hz}, 1 \mathrm{H}$ ), 2.0 (dq, J= 12.2 and $4.8 \mathrm{~Hz}, 1 \mathrm{H}), 2.1$ (m, 4H), 3.6 (m, 2H), 4.0 (m, 3H), 4.4 (dd, $J=1.7$ and 3,5 Hz, 1H), $4.5(\mathrm{~m}, 1 \mathrm{H}), 5.5(\mathrm{~d}, J=16.2$ and $8.1 \mathrm{~Hz}, 1 \mathrm{H}), 5.6(\mathrm{~s}, 1 \mathrm{H}), 6.65(\mathrm{~d}, J=16.2$ $\mathrm{Hz}, 1 \mathrm{H}), 7.3-7.6(\mathrm{~m}, 5 \mathrm{H}) .{ }^{13} \mathrm{C}-\mathrm{NMR}\left(75 \mathrm{MHz}, \mathrm{CDCl}_{3}\right) \delta(\mathrm{ppm})=24.8(\mathrm{t}), 25.5(\mathrm{t}), 31.5(\mathrm{t}), 53.7$ (t), $61.6(\mathrm{t}), 66.8$ (t), 75.9 (d), 101.0 (d), 126.0 (d), 126.4 (d), 128.1 (d), 128.8 (d), 138.4 (s), 141.0 (d), 198.9 (s), 203.4 (s), 246.2 (s).

\section{General procedure for the synthesis of phenols 11}

A THF solution $(20 \mathrm{ml})$ of the alkoxycarbene $\mathbf{1 b}(360 \mathrm{mg}, 1 \mathrm{mmol})$ and alkyne 10 (5 mmol) was kept in a sealed tube at $60^{\circ} \mathrm{C}$ during $8 \mathrm{~h}$. After cooling down, the mixture was filtered through a celite pad and the solvent removed under vacuo $\left(10^{-2}\right.$ torr $)$. The residue was purified by chromatographic column through silica gel to afford phenols 11.

6-tert-Butyl-4-methoxy-2-((S)-2,2-dimethyl-1,3-dioxolan-4-yl)phenol (11a). Yield: 65\%. $\mathrm{R}_{\mathrm{f}}$ (Hex/AcOEt (2:1)): 0.68; ${ }^{1} \mathrm{H}-\mathrm{NMR}\left(300 \mathrm{MHz}, \mathrm{CDCl}_{3}\right): \delta$ (ppm)= 1.39 (s, 9H), 1.50 (s, 3H), 1.60 (s, 3H); 3.74 (s, 3H), 3.93 (dd, $J=8.8$ and $8.3 \mathrm{~Hz}, 1 \mathrm{H}$ ), 4.27 (dd, $J=8.3$ and $6.3 \mathrm{~Hz}, 1 \mathrm{H}$ ), 5.14 (dd, $J=9.1$ and $6.2 \mathrm{~Hz}, 1 \mathrm{H}), 6.38$ (d, $J=2.9 \mathrm{~Hz}, 1 \mathrm{H}), 6.83(\mathrm{~d}, J=2.9 \mathrm{~Hz}, 1 \mathrm{H}), 7.80(\mathrm{~s}, \mathrm{OH}) ;{ }^{13} \mathrm{C}-$ NMR (75 MHz, $\mathrm{CDCl}_{3}$ ): 25.6 (q), 26.5 (q), 29.4 (q), 35.0 (s), 55.6 (q), 69.7 (t), 79.2 (d), 109.7 (s), 110.3 (d), 113.8 (d), 120.8 (s), 139.0 (s), 148.6 (s), 152.0 (s).

6-Butyl-4-methoxy-2-((S)-2,2-dimethyl-1,3-dioxolan-4-yl)phenol (11b). Yield: 60\%. $\mathrm{R}_{\mathrm{f}}$ (Hex/AcOEt (2:1)): 0.70; ${ }^{1} \mathrm{H}-\mathrm{NMR}\left(200 \mathrm{MHz}, \mathrm{CDCl}_{3}\right): \delta(\mathrm{ppm})=0.95$ (t, J= $\left.7.2 \mathrm{~Hz}, 3 \mathrm{H}\right), 1.26-$ 1.68 (m, 4H), 1.51 (s, 3H), 1.60 (s, 3H), 2.59 (t, J= $7.2 \mathrm{~Hz}, 1 \mathrm{H}), 2.61$ (t, J= $7.2 \mathrm{~Hz}, 1 \mathrm{H}), 3.75$ (s, 3H), 3.94 (t, $J=8.7 \mathrm{~Hz}, 1 \mathrm{H}$ ), 4.32 (dd, $J=8.2$ and $6.1 \mathrm{~Hz}, 1 \mathrm{H}), 5.17$ (dd, $J=8.7$ and $6.2 \mathrm{~Hz}, 1 \mathrm{H}$ ), 6.41 (d, $J=3.1,1 \mathrm{H}), 6.68$ (d, $J=3.1,1 \mathrm{H}), 7.50$ (s, 1H).

\section{Acknowledgements}

We acknowledge the financial support received from Ministerio de Ciencia y Tecnología (BQU2001-3855). NP thanks Swiss National Science Fundation for a postdoctoral fellowship and AP acknowledges FICYT (Principado de Asturias) for a predoctoral fellowship.

\section{References}

1. Fischer, E. O; Maasböl, A. Angew. Chem. Int. Ed. Engl. 1964, 3, 580. 
2. For general reviews see: (a) Wulff, W. D. In Comprehensive Organometallic Chemistry II; Abel, E. W.; Stone, F. G. A.; Wilkinson, G. Eds.; Pergamon Press: New York, 1995, Vol. 12, p 469. (b) Harvey, D. F.; Sigano, D. M. Chem. Rev. 1996, 96, 271. (c) Dötz, K. H., Tomuchat, P. Chem. Soc. Rev. 1996, 28, 187. (d) Zaragoza, F. In Metal Carbenes in Organic Synthesis; Wiley-VCH, 1999. (e) Herndon, J. W. Tetrahedron 2000, 56, 1257.

3. Recent review: de Meijere, A.; Schirmer, H.; Duetsch, M. Angew. Chem., Int. Ed. 2000, 39, 3964.

4. Wulff, W. D. Organometallics 1998, 17, 3116.

5. Cooke, M. D.; Fischer, E. O. J. Organomet. Chem. 1973, 56, 279.

6. (a) Barluenga, J.; Muñiz, K.; Tomás, M.; Ballesteros, A.; García-Granda, S. Organometallics 2003, 22, 1756. (b) Barluenga, J.; Muñiz, K.; Ballesteros, A.; Martínez, S.; Tomás, M. ARKIVOC 2002, (v),110.

7. Selected examples using chiral auxiliaries derived from alcohols: From menthol: (a) Dötz, K. H.; Stinner, C. Tetrahedron Asymmetry 1997, 8, 1751. (b) Hsung, R. P.; Wulff, W. D.; Chamberlin, S.; Liu, Y.; Liu, R. Y.; Wang, H.; John, J. F.; Wang, S. L. B.; Rheingold, A. L. Synthesis 2001, 2, 200; From 8-phenylmenthol. (c) Barluenga, J.; Montserrrat, J.; Flórez, J.; García-Granda, S.; Martín, E. Angew. Chem., Int. Ed. 1994, 106, 1451. (d) Hirotaka, K.; Okamura, T.; Akiyama, T. J. Am. Chem. Soc. 2001, 123, 7182. (e) Barluenga, J.; FernándezRodriguez, M. A.; Aguilar, E.; Fernández-Marí, F.; Salinas, A.; Olano, B. Chem. Eur. J. 2001, 7, 3533; From 8-(2-naphtyl)menthol. (f) Barluenga, J.; Ballesteros, A.; Santamaría, J.; Tomás, M. J. Organomet. Chem. 2002, 643-644, 363; From 2-Phenylcyclohexanol: See 7a and 7b; From borneol: See 7a and 7b.

8. Selected examples: (a) Anderson, B. A.; Wulff, W. D.; Rahn, A. J. Am. Chem. Soc. 1993, 115, 4602. (b) Maiorana, S.; Licandro, E.; Capella, L.; Perdicchia, D.; Papagni, A. Pure Appl. Chem. 1999, 71, 1453.

9. (a) Montgomery, J.; Wieber, G. M.; Hegedus, L. S. J. Am. Chem. Soc. 1990, 112, 2814. (b) Hegedus, L. S.; Bates, R.; Soderberg, B. C. J. Am. Chem. Soc. 1991, 113, 923. (c) Hegedus, L. S.; De Weck, G.; D’Andrea, S. J. Am. Chem. Soc. 1988, 110, 2122. (d) Vernier, J. M.; Hegedus, L. S.; Miller, D. B. J. Org. Chem. 1992, 57, 2209.

10. (a) Hegedus, L. S.; Imwinkelreid, R.; Alarid-Sargent, M.; Dvorak, D.; Satoh, Y. J. Am. Chem. Soc. 1990, 112, 1109. (b) Hegedus, L. S.; Schwindt, M. A.; DeLombaert, S.; Imwinkelreid, R. J. Am. Chem. Soc. 1990, 112, 2264.

11. For examples of chiral manganese, iron or molybdenum carbene complexes with central chirality at metal, see: (a) Lugan, N.; Kelley, C.; Terry, M. R.; Geoffroy, G. L.; Rheingold, A. L. J. Am. Chem. Soc. 1990, 112, 3220. (b) Brookhart, M.; Timmers, D.; Tucker, J. R.; Willians, G. D.; Husk, G. R.; Brunner, H.; Hammer, B. J. Am. Chem. Soc. 1983, 105, 6721. (c) Brookhart, M.; Liu, Y.; Goldman, E. W.; Timmers, D. A.; Willians, G. D. J. Am. Chem. Soc. 1991, 113, 927. (d) Gunnoe, T. B.; White, P. S.; Templeton, J. L.; Organometallics 1997, 16, 370. 
12. (a) K. H. Dötz, K. H.; Straub, W.; Ehlenz, R.; Pesele, K.; Meisel, R. Angew. Chem., Int. Ed. 1995, 34, 1856. (b) G. Bühler, M. C. Feiters, R. J. M. Nolte, K. H. Dötz, Angew. Chem., Int. Ed. 2003, 42, 2494.

13. Jackson, D. Y. Synthetic Commun. 1988, 18, 337.

14. (a) Sánchez-Sancho, F.; Valverde, S.; Herradon, B. Tetrahedron: Asymmetry 1996, 7, 3209. (b) Díaz, D. Martín, V. Org. Lett. 2000, 2, 335. (c) Flögel, O. Okala-Amombo, M. G.; Reissig, H. U.; Zahn, G.; Brüdgam, I.; Hartl, H. Chem. Eur. J. 2003, 9, 1405.

15. Aumann, R.; Heinen, H. Chem. Ber. 1987, 120, 537.

16. Wang, H.; Hsung, R. P.; Wulff, W. D. Tetrahedron Lett. 1988, 39, 1849.

17. Dötz, K. H., Angew. Chem., Int. Ed. 1975, 14, 644.

18. For phenol-based dipeptide ligands, see: Akullian, L. C.; Snapper, M. L.; Hoveyda, A. H. Angew. Chem., Int. Ed. 2003, 42, 4244. 